\title{
Modal interaction and higher harmonic generation in a weakly nonlinear, periodic
} mass-spring chain

\section{Frandsen, Niels Morten Marslev; Jensen, Jakob Søndergaard}

\section{Published in:}

Wave Motion

Link to article, DOI:

10.1016/j.wavemoti.2016.09.002

Publication date:

2017

Document Version

Peer reviewed version

Link back to DTU Orbit

Citation (APA):

Frandsen, N. M. M., \& Jensen, J. S. (2017). Modal interaction and higher harmonic generation in a weakly nonlinear, periodic mass-spring chain. Wave Motion, 68, 149-161.

https://doi.org/10.1016/j.wavemoti.2016.09.002

\section{General rights}

Copyright and moral rights for the publications made accessible in the public portal are retained by the authors and/or other copyright owners and it is a condition of accessing publications that users recognise and abide by the legal requirements associated with these rights.

- Users may download and print one copy of any publication from the public portal for the purpose of private study or research.

- You may not further distribute the material or use it for any profit-making activity or commercial gain

- You may freely distribute the URL identifying the publication in the public portal 


\title{
Modal interaction and higher harmonic generation in a weakly nonlinear, periodic mass-spring chain
}

\author{
Niels M. M. Frandsen ${ }^{a, *}$, Jakob S. Jensen ${ }^{b}$ \\ ${ }^{a}$ Section for Solid Mechanics, Department of Mechanical Engineering, Technical University of Denmark, \\ Denmark \\ ${ }^{b}$ Centre for Acoustic-Mechanical Micro Systems, Department of Electrical Engineering, Technical University of \\ Denmark, Denmark
}

\begin{abstract}
Wave propagation in a nonlinear periodic material is investigated, by considering an infinite chain of two-mass unit cells with cubic stiffness nonlinearity. The chain is analyzed using the method of multiple scales, predicting the dispersion shift in the band structure due to nonlinear selfinteraction. The solution further reveals modest higher harmonic generation within the limits of the solution approach, proportional to the strength of nonlinearity and energy level in the chain. The possibility for controlling the higher harmonic generation by changing the distribution of the cubic nonlinearity is investigated. The predictions based on the analytical model are verified by numerical simulations, which also explores the limits of the infinite, analytical model.
\end{abstract}

Keywords: Wave propagation, periodic materials, nonlinear materials, higher harmonic generation.

\section{Introduction}

The fact that periodic materials and structures can exhibit highly interesting dynamic behaviour has been known for, at least, half a century see [1], which also contains a historical description of research before the 1950's. However, the research intensity in periodic materials and structures has increased considerably within the last 25 years, $[2,3,4]$.

One of the most interesting and investigated concepts within material dynamics is the band structure of the material, which relates the spatial and temporal characteristics of the wave through the wavenumber/frequency relation known as the dispersion relation. The band structure of a periodic material can reveal frequency ranges where waves cannot propagate, known as band gaps or stop bands. The position and width of the stop bands depend on system properties, such as periodicity, material properties and geometry, whereby it is possible to design materials or structures with large gaps in desired frequency locations, e.g. by topology optimization [5].

If the material or structure exhibits nonlinear characteristics, additional effects occur, such as intensity dependent dispersion relations, nonlinear interaction and higher harmonic generation. Investigations of the effects of nonlinearity on the band structure have been carried out in e.g. [6] where they also consider the relation to the normal forms of finite systems and the generation of additional waves due to nonlinear interaction between counter-propagating waves. The work by Narisetti and collaborators in $[7,8]$ investigates the band structure for one- and two-dimensional nonlinear periodic structures by a perturbation approach. Nonlinear wave-wave interaction is considered by Manktelow et. al. in [9], where they study the effect of interaction between propagating waves on the dispersion in a cubically nonlinear, homogeneous chain. They consider both the general case as well as the special case of internal resonance where there is a $3: 1$ ratio between wavenumbers and frequencies for the two waves. A similar study was carried out in

*Corresponding author

Email address: nimmfr@mek.dtu.dk (Niels M. M. Frandsen) 
[10] for quadratic nonlinearity, investigating the concept of modal lifetime. Wave-wave interaction for general, one-, two- and three-dimensional, nonlinear periodic lattice materials is investigated in [11], a study that is closely related to the present study.

The first order response for a finite periodic chain with quadratic nonlinearity is considered in [12], and it is verified numerically and experimentally how waves might be generated within the band gap due to nonlinear interaction. Yousefzadeh and Phani consider excitation of a cubically nonlinear, finite, damped chain within a stop band in [13], and illustrate a transmission threshold, above which energy is transported through the chain due to the loss of stability of the periodic solutions.

The present study considers an infinite, cubically nonlinear, inhomogeneous mass-spring chain. The effects of modal interaction on dispersion is investigated, as well as the higher harmonic generation within the chain. Comparing to the wave-interaction analysis in [11], we consider the self-interaction of the presence of both wave-modes rather than the effect of injecting a control wave in the system. The main emphasis of the present study however, is the prediction of higher harmonic generation and its dependency on the nonlinear system parameters. The goal is to investigate, by analytical means, the potential for controlling the higher harmonic generation within such a chain and thereby enable the optimization of the generation of higher harmonic waves.

\section{Model}

An infinite chain of two-mass unit cells illustrated in Figure 1 is considered, where $k_{i}$ and $\Gamma_{i}$ are the linear and nonlinear stiffness coefficients respectively, while $m_{i}$ are the masses in the chain.

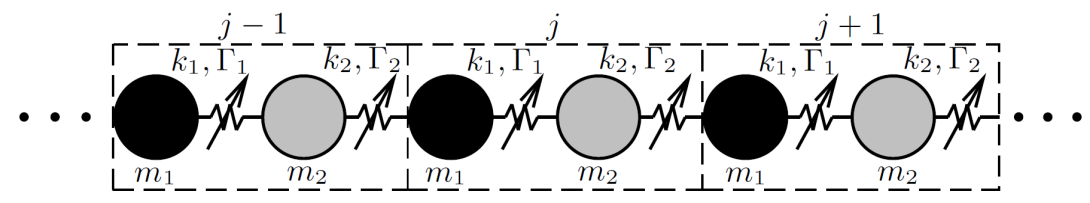

Figure 1: Infinite chain of two-mass unit cells

The governing equations for unit cell $j$ are:

$$
\mathbf{M} \ddot{\mathbf{u}}_{j}+\mathbf{K} \mathbf{u}_{j}+\mathbf{f}_{j}^{L}\left(u_{j-1,2}, u_{j+1,1}\right)+\mathbf{f}_{j}^{N L}\left(\mathbf{u}_{j}, u_{j-1,2}, u_{j+1,1}\right)=\mathbf{0}, \quad \mathbf{u}_{j}=\left[\begin{array}{l}
u_{j, 1}(t) \\
u_{j, 2}(t)
\end{array}\right]
$$

where $u_{j, n}(t)$ is the displacement of mass $n$ in unit cell $j$. Furthermore, $\left.\ddot{(}\right)=\partial^{2} / \partial t^{2}$ and the system matrices and force vectors are given by:

$$
\begin{array}{r}
\mathbf{M =}\left[\begin{array}{cc}
m_{1} & 0 \\
0 & m_{2}
\end{array}\right], \quad \mathbf{K}=\left[\begin{array}{cc}
k_{1}+k_{2} & -k_{1} \\
-k_{1} & k_{1}+k_{2}
\end{array}\right], \quad \mathbf{f}_{j}^{L}\left(u_{j-1,2}, u_{j+1,1}\right)=-k_{2}\left[\begin{array}{c}
u_{j-1,2} \\
u_{j+1,1}
\end{array}\right] \\
\mathbf{f}_{j}^{N L}\left(\mathbf{u}_{j}, u_{j-1,2}, u_{j+1,1}\right)=\left[\begin{array}{c}
\Gamma_{1}\left(u_{j, 1}-u_{j, 2}\right)^{3}+\Gamma_{2}\left(u_{j, 1}-u_{j-1,2}\right)^{3} \\
\Gamma_{1}\left(u_{j, 2}-u_{j, 1}\right)^{3}+\Gamma_{2}\left(u_{j, 2}-u_{j+1,1}\right)^{3}
\end{array}\right] .
\end{array}
$$

The nonlinear equations of motion in Eq. (1) govern the dynamics of the periodic medium illustrated in Figure 1. In Section 3 the wave-characteristics of the infinite chain are investigated by the method of multiple scales, including higher harmonic generation. In Section 4, the higher harmonic predictions of the analytical solution are compared to numerical results. These results are obtained by numerical integration of the governing equations for a finite-length chain that is comparable to the infinite medium. 


\section{Multiple Scales Analysis}

The governing equations for unit cell $j$ in Eq. (1) depend on the motion of the neighbouring cells. For linear periodic systems, the motion of the neighbouring cells can be related to the motion of the considered unit cell through a phase-multiplier, by the Floquet-Bloch theorem [14]. The wave-characteristics of the nonlinear medium are investigated by approximating the nonlinear equations by a set of linear ones, using the method of multiple scales, [15].

The method of multiple scales is a perturbation approach, hence the nonlinear elastic forces are assumed to be much smaller than the linear, i.e.,

$$
\mathbf{M} \ddot{\mathbf{u}}_{j}+\mathbf{K} \mathbf{u}_{j}+\mathbf{f}_{j}^{L}\left(u_{j-1,2}, u_{j+1,1}\right)+\varepsilon \mathbf{f}_{j}^{N L}\left(\mathbf{u}_{j}, u_{j-1,2}, u_{j+1,1}\right)=\mathbf{0}
$$

where the parameter $\varepsilon$ is a book-keeping parameter, indicating the smallness of the nonlinear terms. A uniformly valid expansion of the displacements in unit cell $j$ is made:

$$
\mathbf{u}_{j}(t)=\mathbf{u}_{j}^{(0)}\left(T_{0}, T_{1}\right)+\varepsilon \tilde{\mathbf{u}}_{j}^{(1)}\left(T_{0}, T_{1}\right)+O\left(\varepsilon^{2}\right), \quad T_{k}=\varepsilon^{k} t, \quad\left|\varepsilon \tilde{\mathbf{u}}_{j}^{(1)}\right|=\left|\mathbf{u}_{j}^{(1)}\right|<<\left|\mathbf{u}_{j}^{(0)}\right|
$$

where two new time-scales have been introduced. Inserting in Eq. (3) and setting terms of equal power in $\varepsilon$ to zero yields:

$$
\begin{array}{ll}
\varepsilon^{0}: & \mathbf{M} D_{0}^{2} \mathbf{u}_{j}^{(0)}+\mathbf{K} \mathbf{u}_{j}^{(0)}+\mathbf{f}_{j}^{L,(0)}=0 \\
\varepsilon^{1}: & \mathbf{M} D_{0}^{2} \mathbf{u}_{j}^{(1)}+\mathbf{K} \mathbf{u}_{j}^{(1)}+\mathbf{f}_{j}^{L,(1)}=-2 \mathbf{M} D_{0} D_{1} \mathbf{u}_{j}^{(0)}-\mathbf{f}_{j}^{N L,(0)}
\end{array}
$$

where $D_{k}^{i}=\partial^{i} / \partial T_{k}^{i}$, and terms of order $O\left(\varepsilon^{2}\right)$ and above have been disregarded. The governing, nonlinear equations are now represented by linear equations at two levels of approximation. The functional dependence of the force vectors on the displacements have been left out for brevity. The order $\varepsilon^{0}$ equations in Eq. (5a) are satisfied by a travelling wave solution:

$$
\mathbf{u}_{j}^{(0)}\left(T_{0}, T_{1}\right)=a\left(T_{1}\right)\left[\begin{array}{l}
A_{1} \\
A_{2}
\end{array}\right] e^{i\left(\mu j+\omega T_{0}\right)}+\text { c.c. }=a\left(T_{1}\right) \mathbf{v} e^{i\left(\mu j+\omega T_{0}\right)}+\text { c.c. }
$$

where $a\left(T_{1}\right)$ is a complex amplitude that depends on slow time $T_{1}$, and c.c. denotes complex conjugate. The relative amplitudes of the masses are given by $A_{i}$ while $\mu$ and $\omega$ are the wavenumber and propagating frequency respectively. Imposing the wave-solution in the order $\varepsilon^{0}$ equations and using the Floquet-Bloch theorem, leads to a wavenumber-dependent eigenvalue-problem in $\omega^{2}$ and $\mathbf{v}$. Using the transformation $\mathbf{v}=\mathbf{M}^{-1 / 2} \mathbf{z}$ where $\mathbf{M}^{-1 / 2}=\operatorname{diag}\left[\sqrt{m_{1}} \sqrt{m_{2}}\right]$ and pre-multiplying by $\mathbf{M}^{-1 / 2}$ provides the normalized eigenvalue-problem:

$$
\left(\widetilde{\mathbf{K}}(\mu)-\bar{\omega}^{2} \mathbf{I}\right) \mathbf{z} a\left(T_{1}\right)=\mathbf{0}, \quad \widetilde{\mathbf{K}}(\mu)=\left[\begin{array}{cc}
1 & -\left(c_{1}+c_{2} e^{-i \mu}\right) \\
-\left(c_{1}+c_{2} e^{i \mu}\right) & \beta
\end{array}\right]
$$

where $\bar{\omega}=\omega / \omega_{n}, \omega_{n}^{2}=\left(k_{1}+k_{2}\right) / m_{1}, \beta=m_{1} / m_{2}$ and $c_{i}^{2}=k_{i} /\left(\omega_{n}^{2} \sqrt{m_{1} m_{2}}\right)$. Solving the eigenvalue problem in Eq. (7) provides the eigenvalues and -vectors:

$$
\begin{aligned}
\bar{\omega}_{k}^{2} & =\frac{1}{2}\left(1+\beta \pm \sqrt{(1-\beta)^{2}+4\left(c_{1}^{4}+c_{2}^{4}+2 c_{1}^{2} c_{2}^{2} \cos (\mu)\right.}\right) \\
\mathbf{z}_{k} & =\left[-\frac{2\left(c_{1}^{2}+c_{2}^{2} e^{-i \mu}\right)}{\beta-1 \pm \sqrt{(1-\beta)^{2}+4\left(c_{1}^{4}+c_{2}^{4}+2 c_{1}^{2} c_{2}^{2} \cos (\mu)\right)}} \quad 1\right]^{T}, \quad k=1,2
\end{aligned}
$$

corresponding to the linear propagation frequencies and relative amplitudes of the masses in the two modes supported by the two-mass unit cell. The lower mode $(k=1)$ is traditionally referred 
to as the acoustic mode, while the upper mode $(k=2)$ is referred to as the optical mode. The full solution to the order $\varepsilon^{0}$ equations is:

$$
\mathbf{u}_{j}^{(0)}\left(T_{0}, T_{1}\right)=\sum_{k=1}^{2} a_{k}\left(T_{1}\right) \mathbf{v}_{k} e^{i\left(\mu j+\omega_{k 0} T_{0}\right)}+c . c ., \quad \mathbf{v}_{k}=\mathbf{M}^{-1 / 2} \mathbf{z}_{k}
$$

where $a_{k}\left(T_{1}\right)$ will be determined at the next level of approximation. Inserting the order $\varepsilon^{0}$ solution into Eq. (5b) leads to the order $\varepsilon^{1}$ equations:

$$
\begin{aligned}
\mathbf{M} D_{0}^{2} \mathbf{u}_{j}^{(1)}+\mathbf{K} \mathbf{u}_{j}^{(1)}+\mathbf{f}_{j}^{L, 1} & =\mathbf{q}_{1} e^{i\left(\mu j+\omega_{1} T_{0}\right)}+\mathbf{q}_{2} e^{i\left(\mu j+\omega_{2} T_{0}\right)}+\mathbf{q}_{12} e^{i\left(\mu j+\left(2 \omega_{1}-\omega_{2}\right) T_{0}\right)} \\
& +\mathbf{q}_{21} e^{i\left(\mu j+\left(2 \omega_{2}-\omega_{1}\right) T_{0}\right)}+\mathbf{p}_{12} e^{i\left(3 \mu j+\left(2 \omega_{1}+\omega_{2}\right) T_{0}\right)} \\
& +\mathbf{p}_{21} e^{i\left(3 \mu j+\left(\omega_{1}+2 \omega_{2}\right) T_{0}\right)}+\mathbf{p}_{1} e^{3 i\left(\mu j+\omega_{1} T_{0}\right)}+\mathbf{p}_{2} e^{3 i\left(\mu j+\omega_{2} T_{0}\right)}+\text { c.c. }
\end{aligned}
$$

where the coefficient vectors depend on the complex modal amplitudes, $a_{1}, a_{2}$, and the wavenumber $\mu$. They can be found in Appendix A. Any loading terms in Eq. (10) with functional dependence on the eigenvalues of the linear problem will lead to secular behaviour of the solution and must be eliminated. It is clear that the coefficient vectors $\mathbf{q}_{1}$ and $\mathbf{q}_{2}$ will always lead to secular behaviour. Furthermore, for specific wavenumber/frequency combinations, additional resonant terms can occur. This is the case of internal resonance, where the higher harmonic excites an eigen-mode of the system. This will cause the uniformity of expansion, $\left|\mathbf{u}_{j}^{(1)}\right|<<\left|\mathbf{u}_{j}^{(0)}\right|$, to break down, and should be considered separately. Internal resonance is not a focus of the present paper, hence we will focus on the case where only $\mathbf{q}_{1}$ and $\mathbf{q}_{2}$ are resonant. They will be eliminated by choosing the complex modal amplitudes $a_{k}\left(T_{1}\right)$ appropriately, whereby the nonlinear frequency corrections are obtained.

\subsection{Frequency corrections}

Considering the case away from internal resonance, Eq. (10) reduces to

$$
\mathbf{M} D_{0}^{2} \mathbf{u}_{j}^{(1)}+\mathbf{K} \mathbf{u}_{j}^{(1)}+\mathbf{f}_{j}^{L, 1}=\mathbf{q}_{1} e^{i\left(\mu j+\omega_{1} T_{0}\right)}+\mathbf{q}_{2} e^{i\left(\mu j+\omega_{2} T_{0}\right)}+O H T+\text { c.c. }
$$

where OHT denotes Other Harmonic Terms. Several approaches for determining the solvability condition, eliminating secular behaviour from Eq. (10), exist. Here a straightforward modal approach is used. A modal solution to the order $\varepsilon^{1}$ equations is assumed:

$$
\mathbf{u}_{j}^{(1)}\left(T_{0}, T_{1}\right)=\mathbf{V r}\left(j, T_{0}, T_{1}\right), \quad \mathbf{V}=\left[\mathbf{v}_{1} \mathbf{v}_{2}\right]=\mathbf{M}^{-1 / 2} \mathbf{Z}, \mathbf{r}\left(j, T_{0}, T_{1}\right)=\left[\begin{array}{c}
r_{1}\left(j, T_{0}, T_{1}\right) \\
r_{2}\left(j, T_{0}, T_{1}\right)
\end{array}\right]
$$

where $\mathbf{Z}$ is the modal matrix of orthogonal eigenvectors. Inserting in Eq. (10) and premulitplying by $\mathbf{Z}^{H} \mathbf{M}^{-1 / 2}$ yields

$$
\mathbf{Z}^{H} \mathbf{Z} D_{0}^{2} \mathbf{r}+\mathbf{Z}^{H} \widetilde{\mathbf{K}}(\mu) \mathbf{Z r}=\widehat{\mathbf{q}}_{1} e^{i\left(\mu j+\omega_{1} T_{0}\right)}+\widehat{\mathbf{q}}_{2} e^{i\left(\mu j+\omega_{2} T_{0}\right)}
$$

where superscript ${ }^{H}$ indicates Hermitian transpose while $\widehat{\mathbf{q}}_{i}=\mathbf{Z}^{H} \mathbf{M}^{-1 / 2} \mathbf{q}_{i}$. Since the eigenvectors are orthogonal, the left hand side of Eq. (13) decouples, whereby the system of equations can be written as two separate, modal equations,

$$
D_{0}^{2} r_{k}+\omega_{k}^{2} r_{k}=\widehat{q}_{1 k} e^{i\left(\mu j+\omega_{1} T_{0}\right)}+\widehat{q}_{2 k} e^{i\left(\mu j+\omega_{2} T_{0}\right)}+\text { c.c. }, \quad k=1,2
$$

where the eigenvalues of the homogeneous part are $\omega_{k}^{2}=\mathbf{z}_{k}^{H} \widetilde{\mathbf{K}}(\mu) \mathbf{z}_{k} / \mathbf{z}_{k}^{H} \mathbf{z}_{k}$. The decoupled equations reveal that $\widehat{q}_{11}$ is resonant to the first mode while $\widehat{q}_{22}$ is resonant to the second mode. Thus, the solvability condition is: 


$$
\begin{aligned}
& \widehat{q}_{11}=\mathbf{z}_{1}^{H} \mathbf{M}^{-1 / 2} \mathbf{q}_{1}=0 \\
& \widehat{q}_{22}=\mathbf{z}_{2}^{H} \mathbf{M}^{-1 / 2} \mathbf{q}_{2}=0
\end{aligned}
$$

which can be obtained by the adjoint eigenvector approach from [15] as well. Using the expressions for the coefficient vectors $\mathbf{q}_{1}$ and $\mathbf{q}_{2}$ from Eqs. (A.1) leads to the two complex equations:

$$
\begin{aligned}
& -i 2 \omega_{1} a_{1}^{\prime}-3 a_{1}^{2} \bar{a}_{1} \eta_{1}-6 a_{1} a_{2} \bar{a}_{2} \xi_{12}=0 \\
& -i 2 \omega_{2} a_{2}^{\prime}-3 a_{2}^{2} \bar{a}_{2} \eta_{2}-6 a_{2} a_{1} \bar{a}_{1} \xi_{21}=0
\end{aligned}
$$

where $a_{k}^{\prime}=d a_{k} / d T_{1}$ and the scalars $\eta_{k}$ and $\xi_{k i}$ are defined as:

$$
\eta_{k}=\frac{\mathbf{z}_{k}^{H} \mathbf{M}^{-1 / 2}}{\mathbf{z}_{k}^{H} \mathbf{z}_{k}} \sum_{l, m, n} \mathbf{h}_{l m n} z_{k}^{l} z_{k}^{m} \bar{z}_{k}^{n} \quad \xi_{k i}=\frac{\mathbf{z}_{k}^{H} \mathbf{M}^{-1 / 2}}{\mathbf{z}_{k}^{H} \mathbf{z}_{k}} \sum_{l, m, n} \mathbf{h}_{l m n} z_{k}^{l} z_{i}^{m} \bar{z}_{i}^{n}, \quad \begin{aligned}
& k=1,2 \\
& i=1,2
\end{aligned}
$$

The coefficient vector $\mathbf{h}_{l m n}$ can be found in Table A.3, and $z_{k}^{p}$ is the $p^{\text {th }}$ element of the normalized eigenvector of mode $k$. The complex modal amplitudes are now expressed on polar form, $a_{k}=$ $\frac{\alpha_{k}}{2} e^{i \varphi_{k}}$, where $\alpha_{k}\left(T_{1}\right)$ and $\varphi_{k}\left(T_{1}\right)$ are real-valued functions. Separating the real and imaginary parts leads to four coupled modulation equations governing the slow-time variation of the order $\varepsilon^{0}$ solution.

$$
\begin{aligned}
\omega_{1} \alpha_{1}^{\prime} & =0 \\
\omega_{1} \alpha_{1} \varphi_{1}^{\prime} & =\frac{3}{8} \eta_{1} \alpha_{1}^{3}+\frac{3}{4} \xi_{12} \alpha_{1} \alpha_{2}^{2} \\
\omega_{2} \alpha_{2}^{\prime} & =0 \\
\omega_{2} \alpha_{2} \varphi_{2}^{\prime} & =\frac{3}{8} \eta_{2} \alpha_{2}^{3}+\frac{3}{4} \xi_{21} \alpha_{2} \alpha_{1}^{2}
\end{aligned}
$$

It is noted that the scalars defined in Eq. (17) are entirely real entities. The amplitudes are seen to be constant in slow time, $\alpha_{1}\left(T_{1}\right)=\alpha_{10}$ and $\alpha_{2}\left(T_{1}\right)=\alpha_{20}$, whereby the phase equations are easily integrated.

$$
\begin{aligned}
\varphi_{1}\left(T_{1}\right) & =\frac{3}{8} \frac{\eta_{1}}{\omega_{1}} \alpha_{10}^{2} T_{1}+\frac{3}{4} \frac{\xi_{12}}{\omega_{1}} \alpha_{20}^{2} T_{1}+\varphi_{10}, & & \alpha_{10} \neq 0 \\
\varphi_{2}\left(T_{1}\right) & =\frac{3}{8} \frac{\eta_{2}}{\omega_{2}} \alpha_{20}^{2} T_{1}+\frac{3}{4} \frac{\xi_{21}}{\omega_{2}} \alpha_{10}^{2} T_{1}+\varphi_{20}, & & \alpha_{20} \neq 0
\end{aligned}
$$

Hence, the order $\varepsilon^{0}$ solution is

$$
\begin{aligned}
\mathbf{u}_{j}^{(0)}(t) & =\frac{\alpha_{10}}{2} \mathbf{v}_{1} e^{i \varphi_{1}} e^{i\left(\mu j+\omega_{1} t\right)}+\frac{\alpha_{20}}{2} \mathbf{v}_{2} e^{i \varphi_{2}} e^{i\left(\mu j+\omega_{2} t\right)}+c . c . \\
& =\alpha_{10} \cos \left(\mu j+\widetilde{\omega}_{1} t+\varphi_{10}\right)+\alpha_{20} \cos \left(\mu j+\widetilde{\omega}_{2} t+\varphi_{20}\right)
\end{aligned}
$$

where the original time-variable has been reintroduced. The parameters $\alpha_{k 0}$ and $\varphi_{k 0}$ depend on the initial conditions. The first-order corrected frequencies $\widetilde{\omega}_{k}$ are:

$$
\begin{aligned}
& \widetilde{\omega}_{1}=\omega_{1}+\varepsilon\left(\frac{3}{8} \frac{\eta_{1}}{\omega_{1}} \alpha_{10}^{2}+\frac{3}{4} \frac{\xi_{12}}{\omega_{1}} \alpha_{20}^{2}\right)=\omega_{1}+\varepsilon\left(\Delta \omega_{11}+\Delta \omega_{12}\right) \\
& \widetilde{\omega}_{2}=\omega_{2}+\varepsilon\left(\frac{3}{8} \frac{\eta_{2}}{\omega_{2}} \alpha_{20}^{2}+\frac{3}{4} \frac{\xi_{21}}{\omega_{2}} \alpha_{10}^{2}\right)=\omega_{2}+\varepsilon\left(\Delta \omega_{21}+\Delta \omega_{22}\right)
\end{aligned}
$$


revealing that for the two-mass chain, the presence of both waves affects the propagation frequencies for both modes through the coupling terms $\Delta \omega_{12}$ and $\Delta \omega_{21}$. Figure 2 illustrates the effect of modal interaction for an example chain with the parameters given in Table 1, in the normalized range $\bar{\mu}=\mu / \pi, \bar{\omega}=\omega / \omega_{n}$, covering the first Brillouin zone. The amplitudes have been chosen

Table 1: Model parameters

\begin{tabular}{cccccc|cccc}
\hline$m_{1}$ & $m_{2}$ & $k_{1}$ & $k_{2}$ & $\Gamma_{1}$ & $\Gamma_{2}$ & $\beta$ & $\omega_{n}$ & $c_{1}$ & $c_{2}$ \\
1 & 2 & 1 & 5 & \pm 0.05 & \pm 0.05 & 0.5 & 2.45 & 0.34 & 0.77 \\
\hline
\end{tabular}

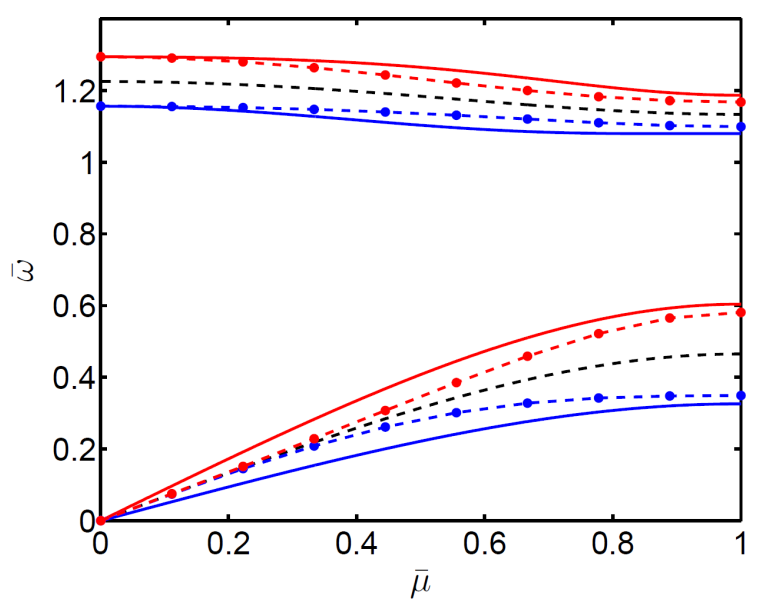

Figure 2: Band structure, $\alpha_{10}=2, \alpha_{20}=2$. Dashed lines: linear dispersion, solid lines: nonlinear corrections (softening and hardening), dash-dot lines: excluding modal interaction

relatively large to exaggerate the results for easier visual inspection. The dashed black lines represent the linear dispersion, while the dash-dot lines represent the case where there is no modal interaction, obtained by imposing the waves separately. The solid lines represent the dispersion when accounting for the modal interaction, as given by Eqs. (21). The curves below the linear dispersion correspond to pure softening nonlinearity $\Gamma_{1}=\Gamma_{2}=-0.05$, while the curves above correspond to pure hardening nonlinearity $\Gamma_{1}=\Gamma_{2}=0.05$. The figure illustrates that modal interaction between the two supported modes, does indeed alter the band structure of the nonlinear material. This was expected from the results of wave-interaction from [11], however the present model accounts for the full modal solution in a two-mass nonlinear periodic chain. It is noted that the largest effect of including modal interaction is fund near the middle of the first Brillouin zone, which means that the largest frequency shift, and perhaps even the gap limits, might not be found on the edges of the first zone. This should be kept in mind if doing a systematic design, or simply, if considering $2 \mathrm{D}$-structures, where the band structure is usually determined by spanning the edge of the first Brillouin zone.

\subsection{First order response}

The approximate analytical response $\mathbf{u}_{j}(t)$ is obtained as the sum of the homogeneous solution to the order $\varepsilon^{0}$ equations and the particular solutions to the order $\varepsilon^{1}$ equations. The homogeneous solution is already known, and determining the particular solutions means solving the equations:

$$
\begin{aligned}
\mathbf{M} D_{0}^{2} \mathbf{u}_{j}^{(1)}+\mathbf{K} \mathbf{u}_{j}^{(1)}+\mathbf{f}_{j}^{L, 1} & =\mathbf{q}_{12} e^{i\left(\mu j+\left(2 \omega_{1}-\omega_{2}\right) T_{0}\right)}+\mathbf{q}_{21} e^{i\left(\mu j+\left(2 \omega_{2}-\omega_{1}\right) T_{0}\right)}+\mathbf{p}_{12} e^{i\left(3 \mu j+\left(2 \omega_{1}+\omega_{2}\right) T_{0}\right)} \\
& +\mathbf{p}_{21} e^{i\left(3 \mu j+\left(\omega_{1}+2 \omega_{2}\right) T_{0}\right)}+\mathbf{p}_{1} e^{3 i\left(\mu j+\omega_{1} T_{0}\right)}+\mathbf{p}_{2} e^{3 i\left(\mu j+\omega_{2} T_{0}\right)}+\text { c.c. }
\end{aligned}
$$


We will focus on higher harmonic generation from the acoustic branch when it exists alone, i.e. $\alpha_{20}=0$. Hence, Eqs. (22) reduces to

$$
\mathbf{M} D_{0}^{2} \mathbf{u}_{j}^{(1)}+\mathbf{K} \mathbf{u}_{j}^{(1)}+\mathbf{f}_{j}^{L, 1}=-\frac{\alpha_{10}^{3}}{8} \mathbf{b}_{3} e^{3 i\left(\mu j+\widetilde{\omega}_{1} t\right)}+c . c .
$$

where $\mathbf{b}_{3}=\sum_{l, m, n} \mathbf{g}_{l m n} z_{1}^{l} z_{1}^{m} z_{1}^{n}$ and the amplitude and phase relations from Eqs. (18) have been utilized. The vector $\mathbf{g}_{l m n}$ can be found in Table A.4. Inserting a travelling wave solution in Eq. (23), $\mathbf{u}_{\mathrm{part}}=\mathbf{y} e^{3 i\left(\mu j+\widetilde{\omega}_{1} T_{0}\right)}+$ c.c., and applying the Floquet-Bloch theorem, leads to the algebraic system of equations:

$$
\left(\mathbf{K}(3 \mu)-\left(3 \widetilde{\omega}_{1}\right)^{2} \mathbf{M}\right) \mathbf{y}=-\frac{\alpha_{10}^{3}}{8} \mathbf{b}_{3}
$$

which can be solved for the coefficient vector $\mathbf{y}$. The total, approximate response is then

$$
\begin{aligned}
\mathbf{u}_{j}(t)=\mathbf{u}_{j}^{(0)}(t)+\mathbf{u}_{j}^{(1)}(t) & =\frac{1}{2} \alpha_{10} \mathbf{v}_{1} e^{i\left(\mu j+\widetilde{\omega}_{1} t\right)}-\frac{1}{8} \alpha_{10}^{3}\left(\mathbf{K}(3 \mu)-\left(3 \widetilde{\omega}_{1}\right)^{2} \mathbf{M}\right)^{-1} \mathbf{b}_{3} e^{3 i\left(\mu j+\widetilde{\omega}_{1} t\right)}+c . c . \\
& =\alpha_{10} \mathbf{v}_{1} \cos \left(\mu j+\widetilde{\omega}_{1} t\right)-\frac{1}{4} \alpha_{10}^{3} \mathbf{y} \cos \left(3\left(\mu j+\widetilde{\omega}_{1} t\right)\right) .
\end{aligned}
$$

With the first order response determined, we will investigate higher harmonic generation from an acoustic wave at four different wavenumber/frequency points on the linear acoustic dispersion curve, given in Table 2. The four points represent four distinct regimes of the higher harmonic.

Table 2: Dispersion points

\begin{tabular}{c|cccc}
\hline $\bar{\mu}$ & 0.10 & 0.20 & 0.40 & 0.66 \\
$\bar{\omega}_{1}(\bar{\mu})$ & 0.07 & 0.13 & 0.26 & 0.39 \\
\hline
\end{tabular}

They are illustrated in Figure 3, where identical markers correspond to the fundamental and higher harmonic respectively and the second Brillouin-zone has been included to illustrate the higher harmonic of the last wavenumber/frequency point. For the first, long-wave limit point (blue plus-sign), the higher harmonic is very close to the linear dispersion curve. Hence, it represents a limit where the analytical model might not be valid since internal resonance could be an issue, as investigated for a mono-atomic model in [9]. The results of the mentioned reference however, indicate only modest effects of internal resonance on dispersion in the long-wave limit, but the possible self-excitation from resonant higher harmonic loading is the focus here. The second point (red cross) corresponds to the higher harmonic being away from the dispersion branch, however still within the acoustic pass-band, while the third point (magenta asterisk) has the higher harmonic within the stop-band. For the fourth point (black star), the higher harmonic falls within the optical pass-band, i.e. the higher harmonic is within the pass-band of the "nonexcited" mode. It is noted that the actual loading frequency of the order $\varepsilon^{1}$ equations will be the nonlinear acoustic frequency, $\widetilde{\omega}_{1}$, however for the purpose of identifying the four described regimes of wavenumber/frequency points, the linearized points will suffice. The higher harmonic generation will be investigated for varying nonlinear stiffness-contrast, $\Gamma_{1} / \Gamma_{2}$, considering four fixed values of $\Gamma_{2}=(0.001,0.005,0.01,0.05)$. In order to be able to compare the results from the four wavenumber/frequency points, the four configurations are investigated for the same input energy, defined as the maximum kinetic energy of the linear response:

$$
E_{0}=\left|E_{\text {kin }}^{(0)}\right|=\left|\frac{1}{2} \dot{\mathbf{u}}_{j}^{T} \mathbf{M} \dot{\mathbf{u}}_{j}\right|=\frac{1}{2} \widetilde{m}_{1}\left(\alpha_{10} \omega_{1}\right)^{2}
$$

where $\widetilde{m}_{1}=\Re\left(\mathbf{z}_{1}^{T}\right) \Re\left(\mathbf{z}_{1}\right)$ is the modal mass of the acoustic mode. The higher harmonic generation is quantified in terms of the ratio between the third and first harmonic, i.e. 


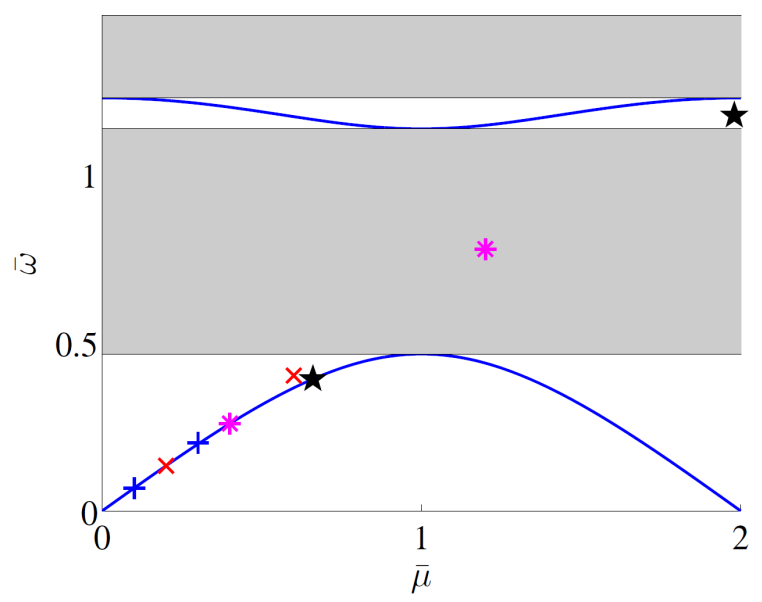

Figure 3: Investigated $\bar{\mu}$-values. Blue plus-signs: Long-wave limit, red crosses: higher harmonic in acoustic pass band, magenta asterisks: higher harmonic in stop band, black stars: higher harmonic in optical pass band.

$$
\left|\frac{A_{3}}{A_{1}}\right|=\frac{\left|\mathbf{u}_{j}^{(1)}\right|}{\left|\mathbf{u}_{j}^{(0)}\right|} .
$$

Figure 4 illustrates how the higher harmonic generation varies with nonlinear stiffness contrast for the four wavenumber/frequency points from Table 2 and input energy $E_{0}=0.1$.

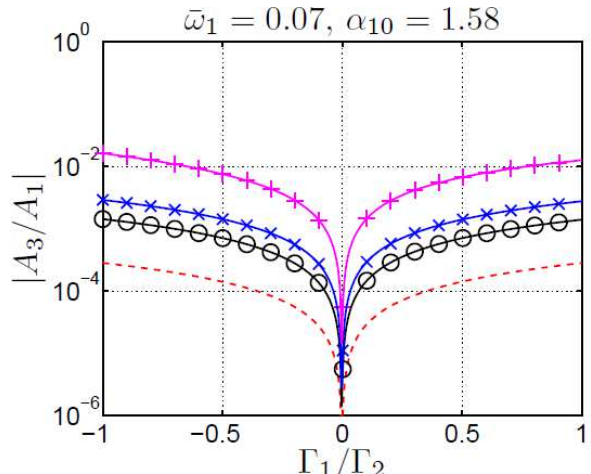

(a)

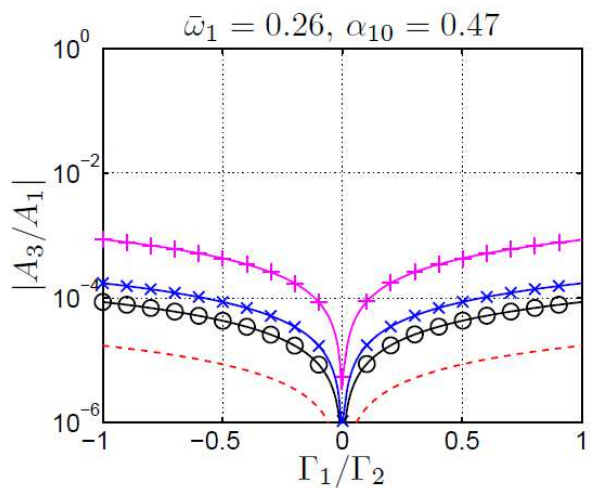

(c)

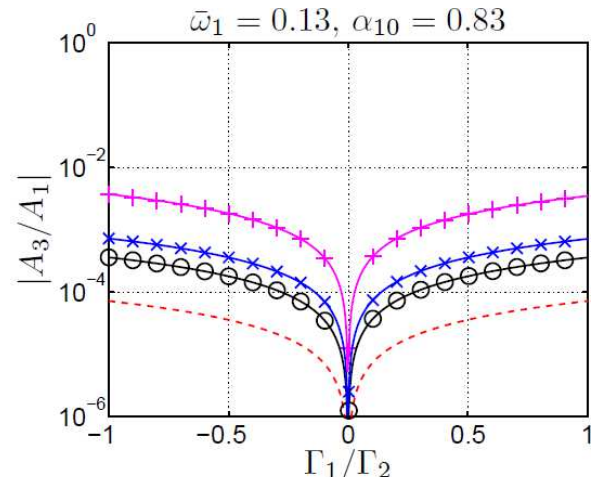

(b)

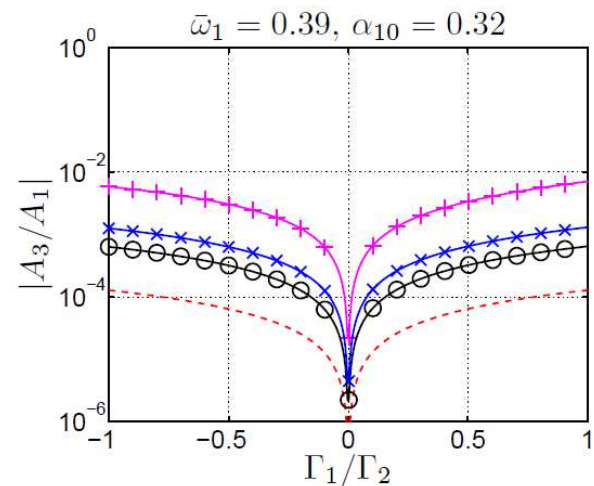

(d)

Figure 4: Effect of nonlinear stiffness ratio, $E_{0}=0.1$. Dashed red line: $\Gamma_{2}=0.001$, black circles: $\Gamma_{2}=0.005$, blue crosses: $\Gamma_{2}=0.01$, magenta plus-signs: $\Gamma_{2}=0.05$. 
It is clear that larger nonlinear coefficients lead to a larger level of higher harmonic generation. The variation with nonlinear stiffness ratio is seen to be almost symmetric around zero, i.e. the higher harmonic generation appears to be fairly insensitive to whether the chain is purely hardening or periodically softening/hardening. Furthermore it can be seen that higher harmonic generation is lowest when it falls within a stop-band, illustrated by Figure $4(\mathrm{C})$, and that it is largest when it is close to a dispersion branch, as illustrated by Figure 4(A). Finally it is noted that, not surprisingly, a much smaller amplitude produces the same level of kinetic energy as the frequency increases from $\bar{\omega}_{1}=0.07$ to $\bar{\omega}_{1}=0.39$.

The maximal higher harmonic generation illustrated in Figure 4 is approximately two orders of magnitude smaller than the input wave, $\max \left(A_{3} / A_{1}\right) \approx 0.01$, i.e. the perturbation assumption of the solution seems to be fulfilled. Figure 5 illustrates the development with nonlinear stiffness ratio for the input energy level $E_{0}=1$.

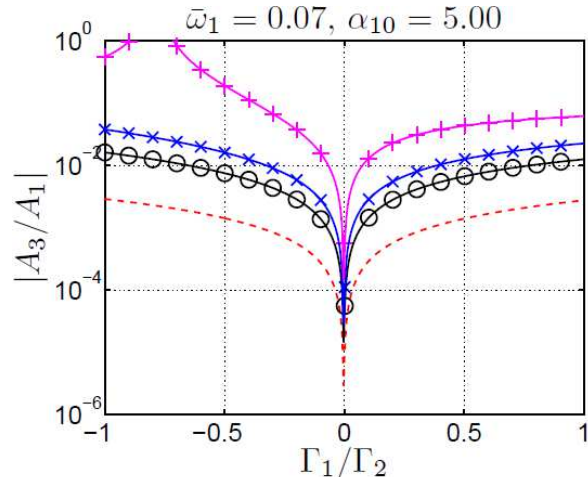

(a)

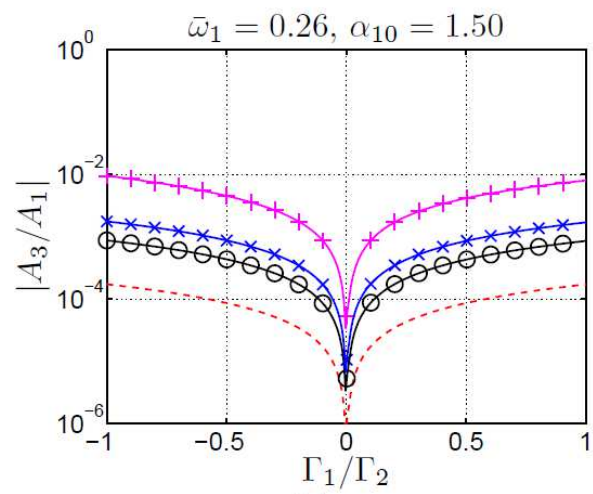

(c)

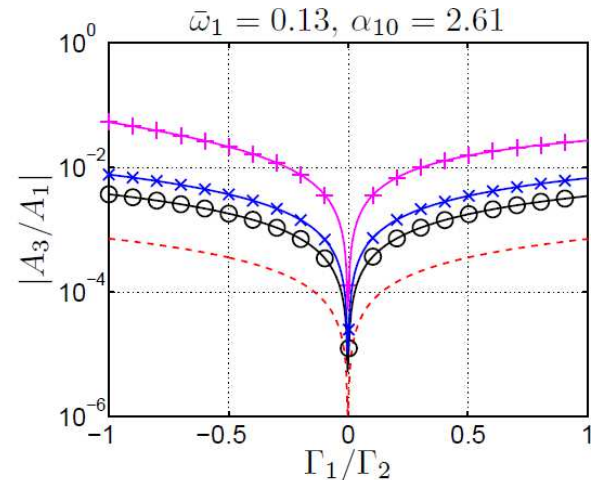

(b)

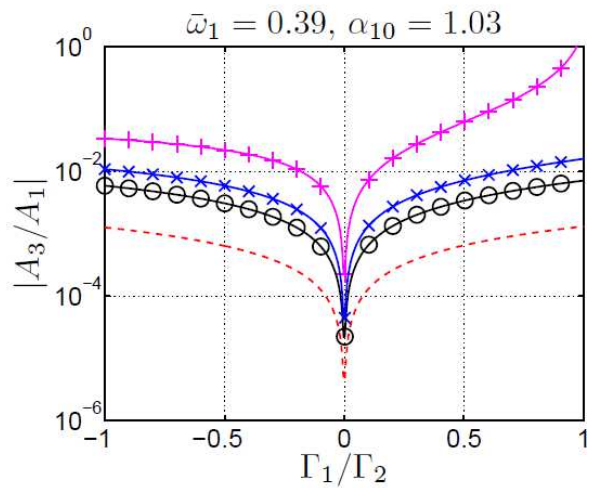

(d)

Figure 5: Effect of nonlinear stiffness ratio, $E_{0}=1$. Dashed red line: $\Gamma_{2}=0.001$, black circles: $\Gamma_{2}=0.005$, blue crosses: $\Gamma_{2}=0.01$, magenta plus-signs: $\Gamma_{2}=0.05$.

The first thing that is noticed, is the appearance of resonance-like peaks for $\bar{\omega}_{1}=0.07$ and $\bar{\omega}_{1}=0.39$. These are the result of internal resonance, and will be discussed further in Section 3.2.1. Aside from these peaks, the general level of higher harmonic generation has increased by approximately one order of magnitude, same as the input energy. This direct scaling of output with input is a result of the approximate solution method, and will only be applicable for weak nonlinearities, i.e. when the nonlinear elastic forces in Eq. (1) are much smaller than the linear ones and as long as the uniformity of the expansion in Eq. (4) is kept. Hence, when the amplitude of the higher harmonic becomes comparable to that of the fundamental harmonic, the model stops producing reliable results. This limitation is illustrated further in Section 4. 


\subsubsection{Internal resonance}

As seen in Figure 5, certain parameter combinations lead to resonance-type behaviour of the order $\varepsilon^{1}$ response. This violates the uniformity of expansion assumption, and consequently the model breaks down for these parameter combinations. However, as mentioned previously, the analysis does not account for internal resonance, the case where the higher harmonic excites an eigen-mode for the system. While the problem is not explicitly revealed by considering the dispersion alone, see Figure 2, the analysis of higher harmonic generation reveals the model breakdown. Figure 6 shows the band-diagram for the infinite chain at the two identified problematic parameter combinations.

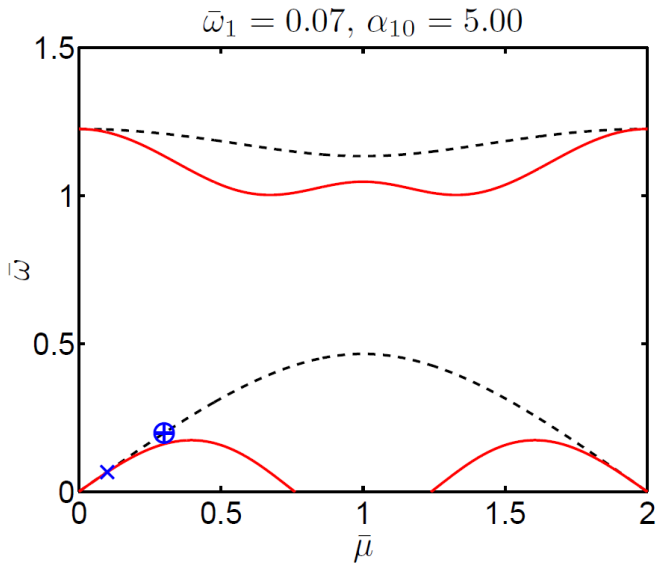

(a)

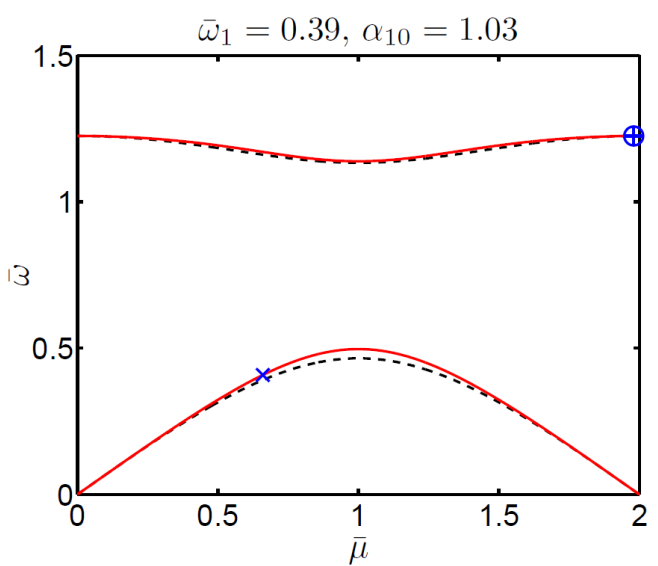

(b)

Figure 6: Internal resonance illustration, $E_{0}=1$. Dashed line: Linear dispersion, solid line: Nonlinear dispersion, blue cross: $\widetilde{\omega}_{1}(\mu) / \omega_{n}$, blue plus-sign: $3 \widetilde{\omega}_{1}(\mu) / \omega_{n}$, blue circle: $\bar{\omega}_{1,2}(3 \mu)$

It can be seen that the higher harmonic $3 \widetilde{\omega}_{1}$ falls exactly on the linear dispersion curve, essentially exciting the system at an eigenvalue. This leads to secular behaviour that should be avoided by imposing specific solvability conditions at the particular frequency ratios leading to internal resonance. This, however, is outside the scope of this work. It is noted that, for $\bar{\omega}_{1}=0.07$ the pertubative nature of the solution approach is clearly violated, since the frequency correction is by no means "small". This cannot be seen for the $\bar{\omega}_{1}=0.39$ case however, illustrating the potential danger of relying strictly on the analytical model, especially when only a partial range of $\bar{\mu}$ is considered. The points of singularity however, can be predicted directly from the dispersion curves so, if remembered, the limits of the analysis method can be evaluated quite directly.

\section{Numerical Analysis}

The approximate response obtained by the analytical model is compared to the numerically obtained response of a finite-length chain. This is done to investigate the validity of the prediction of higher harmonic generation from the analytical model.

\subsection{Model}

The numerical model consists of a finite number of unit cells $N_{\text {cell }}=878$, providing 1756 DOFs. The chain is loaded at the left end by a harmonic force that ramps up from zero over the course of 8 loading periods, i.e.,

$$
F(t)=F_{0}\left(1-e^{-\zeta_{\mathrm{ramp}} t}\right) \sin (\Omega t)
$$

where $\zeta_{\text {ramp }}$ is determined by

$$
\zeta_{\text {ramp }}=-\frac{\ln (1-0.99)}{8 \frac{2 \pi}{\Omega}}
$$


i.e., such that the parenthesis in Eq. (28) is equal to 0.99 after 8 loading periods. In order to avoid any transients in the response, the first part of the chain is (lightly) damped. The damping is imposed by adding viscous dampers between the masses, for a number of unit cells corresponding to five wavelengths of a linear acoustic wave with frequency $\Omega$. The viscous damping is then decreased exponentially over a distance corresponding to five more wavelengths of the acoustic wave to avoid any effects of a discontinuous change in damping properties. The application of the load and damping to the chain is illustrated in Figure 7.

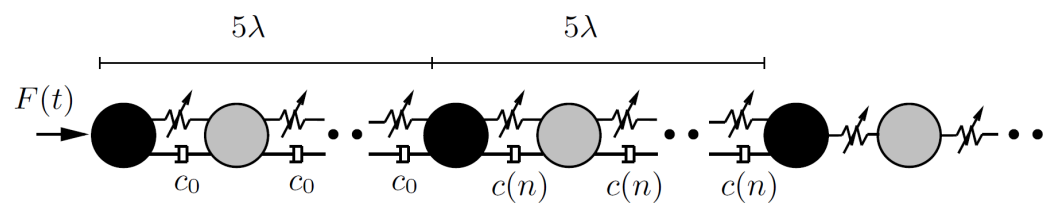

Figure 7: Chain with applied damping and load

The wavelength of the acoustic wave is $\lambda\left(\Omega=\omega_{1}\right)$, the constant damping coefficient is $c_{0}=0.01 \cdot \omega_{n}^{2} \max \left(m_{1}, m_{2}\right)=0.06$ and $c(n)$ is the exponentially decreasing damping coefficient, given by $c(n)=c_{0} e^{-\zeta_{d} n}$ where $n$ is the mass-number and $\zeta_{d}$ is determined such that the exponential function is equal to 0.01 after 5 wavelengths.

\subsection{Higher harmonic generation}

The finite chain is investigated for the same four frequencies as in Sec. 3.2, i.e., $\bar{\Omega}=\Omega / \omega_{n}=\bar{\omega}_{1}$, given in Table 2. The response of the finite chain is calculated for increasing values of the load amplitude $F_{0}$. The simulation time for each simulation is chosen as the travel time for an acoustic wave from the left to the right end of a corresponding linear chain. This simulation time is chosen to avoid reflections from the right boundary in the investigated signals. The numerical simulations will be carried out for a chain with the material parameters given in Table 1 . The numerically obtained response signals of the finite chain are processed by Fourier transforms, in order to obtain the spectral properties as well as the amplitudes of the different harmonics in the signal. The Fourier transforms are done in both the time and space domain, to obtain the actual wavenumber/frequency content of the wave travelling through the chain. The temporal Fourier transform is done for a specific mass, considering only the second half of the simulation time to ensure that a fully developed wave has reached the mass before beginning the transform. The time signal is additionally processed by cutting off any "partial periods" at either end of the signal, so that it is approximately periodic. The signal has further been windowed by a scaled Hannwindow such that the energy is conserved in the frequency spectrum, ensuring correct amplitude information. A similar window has been used for the spatial Fourier transform. Figure 8(a) shows how an excited wave travels through the chain by four snapshots in normalized time $\bar{t}=t / t_{\text {final }}$, where the dashed, vertical black line indicates where the damping stops and the dotted, vertical magenta lines indicate the domain processed by the spatial Fourier transform. Figure 8(b) is the temporal Fourier transform of the mass marked by the red dot in Figure 8(a). The simulation is carried out for the normalized wavenumber $\bar{\mu}=0.2$ and load amplitude $F_{0}=0.25$.

Considering Figure 8(b), the higher harmonic generation is evident from the peak at $3 \bar{\omega}$. This higher harmonic generation from the numerical model will be compared to what is obtained by the analytical model described in Sec. 3.2. The analytical model requires information about the amplitude of the fundamental wave, $\alpha_{10}$, as well as the wavenumber of the wave. The wavenumber is obtained directly from the spatial Fourier transform of the domain illustrated in Figure 8(a). Figure 9 shows the transform at the final time $t_{\text {final }}$, illustrating a peak at the expected normalized wavenumber $\bar{\mu}=0.2$.

It should be noted that the wavenumber measured in the chain shifts slightly with nonlinearity, hence we use the measured wavenumber as input for the analytical calculations.

The amplitude of the fundamental wave is obtained from the temporal Fourier transform. It is related to the analytical amplitude $\alpha_{10}$ through the analytical order $\varepsilon^{0}$ solution when only the 


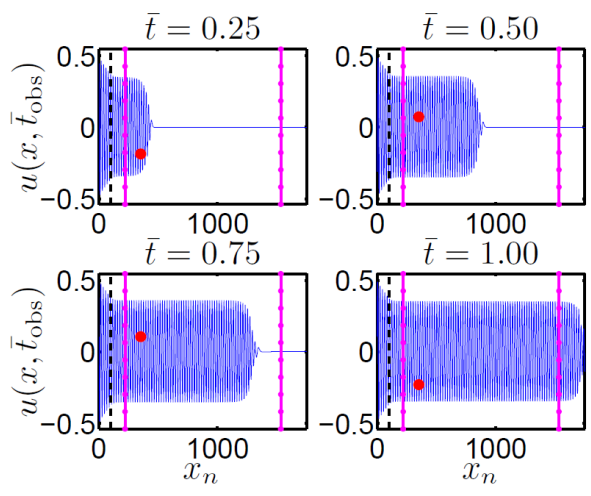

(a)

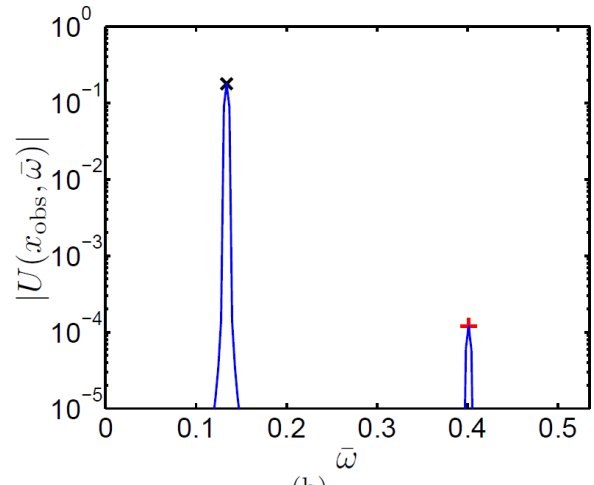

(b)

Figure 8: Example simulation for $\bar{\mu}=0.2$ and $F_{0}=0.25$. Black cross: $\bar{\Omega}$. Red plus-sign: $3 \bar{\Omega}$.

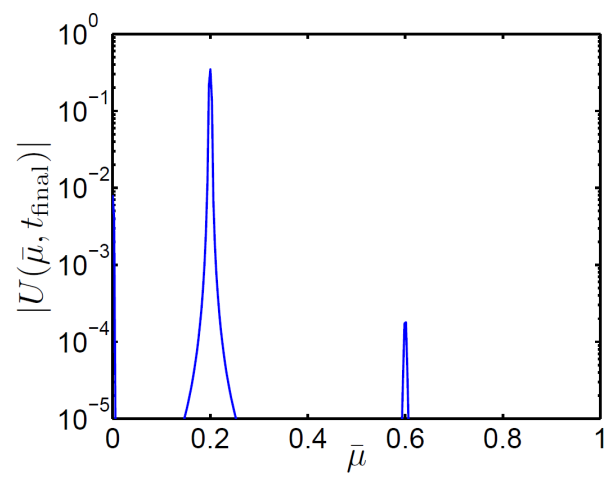

Figure 9: Spatial transform.

acoustic wave is present,

$$
\mathbf{u}_{j}=\frac{1}{2} \alpha_{10}\left(\mathbf{v}_{1} e^{i\left(\mu j+\widetilde{\omega}_{1} T_{0}\right)}+\overline{\mathbf{v}}_{1} e^{-i\left(\mu j+\widetilde{\omega}_{1} T_{0}\right)}\right)
$$

where an overbar means complex conjugate. The max displacement in the unit cell is given by

$$
u_{j, \max }=\alpha_{10} \max \left(\Re\left(\mathbf{v}_{1}\right)\right)
$$

which can be compared directly to the numerically obtained amplitude of the fundamental harmonic, $A_{1}$. Hence, the analytical amplitude $\alpha_{10}$ is obtained from the numerical simulation by

$$
\alpha_{10}=\frac{A_{1}}{\max \left(\Re\left(\mathbf{v}_{1}\right)\right)}
$$

whereby the analytical model can be compared to the numerical model. Figure 10 compares the higher harmonic generation predicted by the analytical and numerical model respectively for the four loading frequencies given in Table 2 .

The crosses and plus-signs represent the numerical and analytical results for measured amplitudes and wavenumbers respectively. The dashed black line approximate the continuous analytical prediction of the higher harmonic generation when the wavenumber is approximated as as the wavenumber corresponding to the input frequency on the linear acoustic dispersion curve rather than the measured wavenumber. The figure illustrates that the analytical model generally predicts the higher harmonic generation well for lower input energies. As the input energy increases, so does the higher harmonic generation, and after a certain threshold, the analytical model stops being accurate. In particular, the long-wave limit case where the higher harmonic is near the acoustic dispersion curve, the results from the analytical model is seen to deteriorate as the input 


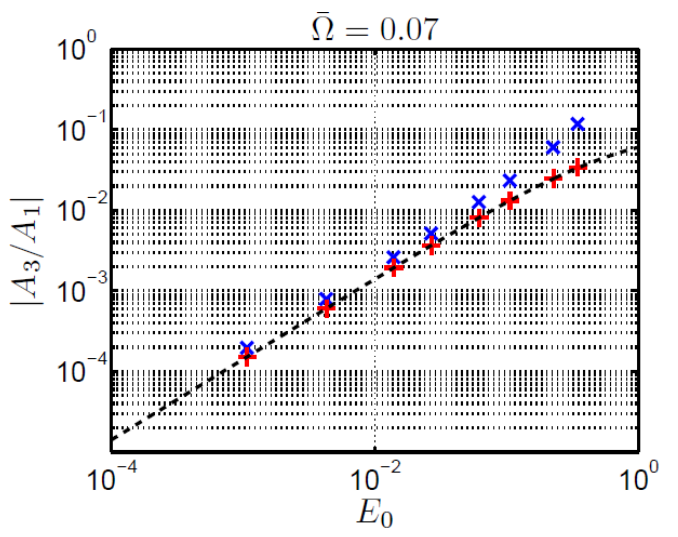

(a)

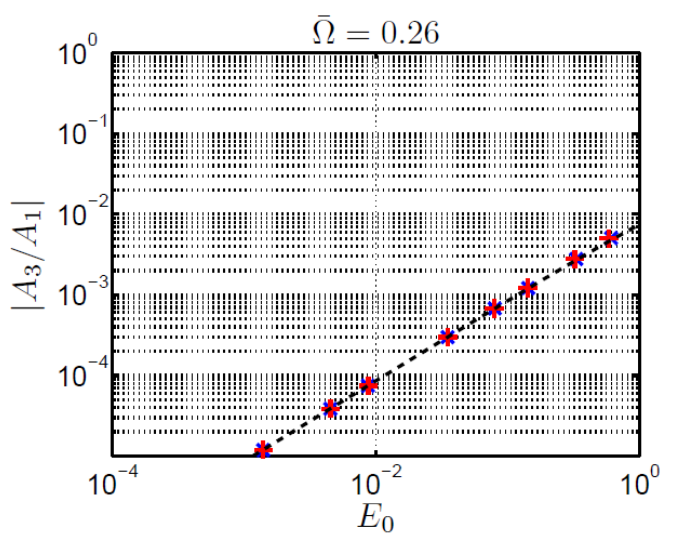

(c)

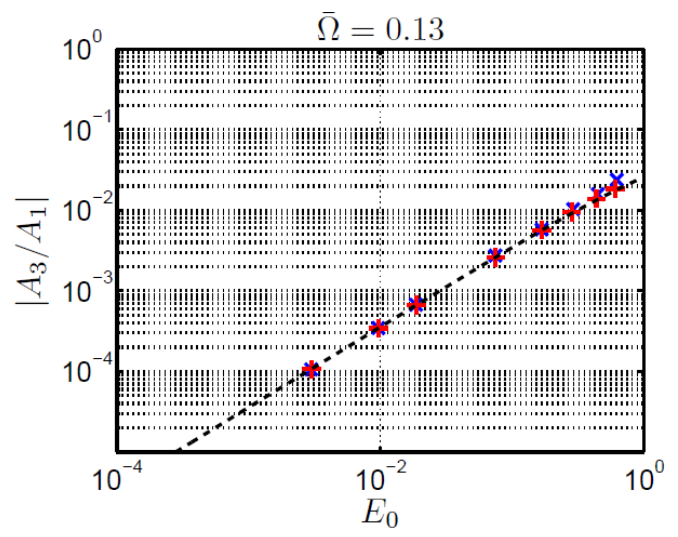

(b)

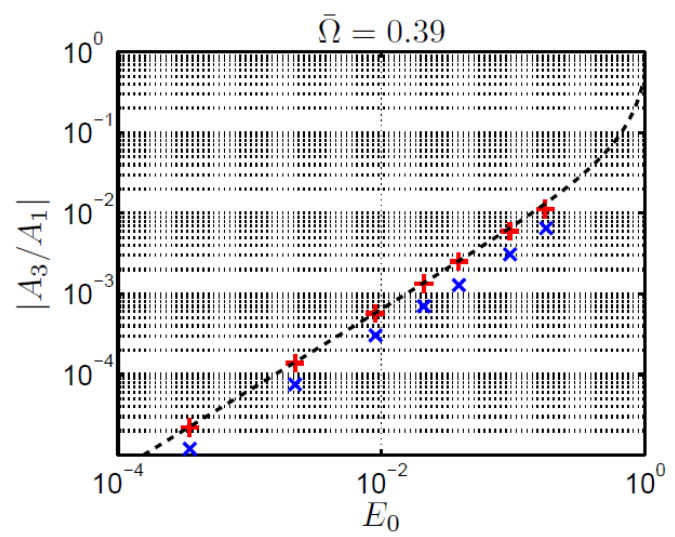

(d)

Figure 10: Higher harmonic generation. Blue crosses: Numerical simulation, red plus-signs: Analytical comparison, Dashed black line: Analytical approximation

energy $E_{0}$ approaches 1 . The analytical predictions are noted to be larger than the numerical results for the investigated energy spectrum in the case with the higher harmonic in the optical pass band. Hence, it appears the the analytical model reaches its limits of accuracy before the internal resonance issue arises, since this is when the analytical curve starts to bend in Figure 10(d). This means that if the prediction of a very large energy transfer from the fundamental to the higher harmonic is desired, a different method than the method of multiple scales should be employed to quantitatively capture the generation. This result was expected, since the method is a perturbation approach, assuming that the order $\varepsilon^{1}$ solution is much smaller than the order $\varepsilon^{0}$ solution, however the results indicate that the qualitative trend can still be captured at low energy levels. Comparing the higher harmonic generation across different loading frequencies, it appears to depend on the input energy in a very similar manner, that is, the slope of the curves are similar. The long-wave limit is seen to generate the largest higher harmonic amplitude, while the loading frequency with the higher harmonic in the band gap is seen to generate the lowest, as seen in the analytical case as well. It is noted that the linear "approximation" of the wavenumber only has a significant effect for the largest loading frequency, at the largest input energies, which is supported by the notion that the shift in wavenumber is a function of nonlinearity.

Finally, the explanation for the numerical points being below the analytical points for $\bar{\Omega}=0.39$ is provided, along with an illustration of the limits of the analytical model. Figure 11 presents the frequency spectra for the final point in Figure 10(d) as well as for a point with an even larger input force, which was not included in the comparison, because the analytical model is clearly not representative any longer, neither is the interpretation of "input energy" as the maximal kinetic energy in the linear acoustic mode. This can be seen clearly by comparing the two spectra, where 
the spectrum for the higher input force actually has a lower "fundamental" harmonic amplitude, but a much wider spectrum, i.e., the energy is dispersed among a greater range of frequencies. The energy dispersion almost looks continuous, compared to Figure 11(a) where distinct frequencies are still seen to carry the energy of the system.

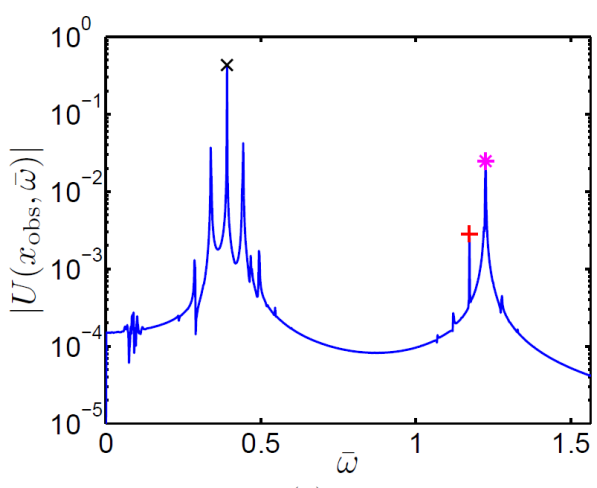

(a)

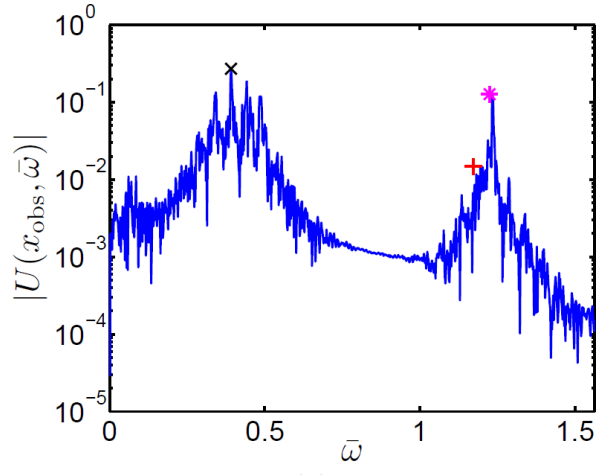

(b)

Figure 11: Frequency spectra for large input energies at $\bar{\Omega}=0.39$. Left panel: $F_{0}=2$, right panel $F_{0}=3$.

The magenta asterisks are the limit frequencies of the optical pass band, which are points with a horizontal tangent on the dispersion curve, i.e., points with zero group velocity. This standing wave point on the dispersion curve is where the resonances of the finite system will gather since the least energy is required for motion at these wavenumbers. Hence, the spectra in Figure 11 illustrates that the energy is transferred from the higher harmonic to the nearby frequency with zero group velocity. This is actually what occurs for all the numerical simulations below the analytical predictions in Figure 10(d), which indicates the relevance of analyzing the finite system at hand rather than a constituent material.

\section{Conclusions}

The present paper investigates the effect of modal interaction on the dispersion of a weakly nonlinear, periodic mass-spring chain. Using the method of multiple scales, the band structure is determined, illustrating that modal interaction increases the frequency shift of the dispersion curve, in particular around the middle of the first Brillouin zone. Hence, the increase in waveenergy in the chain due to the presence of both wave-modes amplifies the intensity dependent effects in the band structure.

Furthermore, higher harmonic generation from an acoustic wave is investigated, analytically and numerically. The higher harmonic generation is seen to depend directly on the input energy as well as the strength of the nonlinearity. Moreover, it is shown that when the higher harmonic falls within a stop band, the generation is approximately one order of magnitude smaller than when it falls within a pass band. Additionally, higher harmonic generation appears to be larger in the long-wave limit. The analytical model is shown to produce reliable results at low energies, and away from internal resonance points. At internal resonance, the analytical model breaks down and predicts infinite order $\varepsilon^{1}$ response. The general accuracy of the analytical model deteriorates at lower energy levels than is required for internal resonance to occur, so the implications are not too severe. However, if one is to a apply a systematic design approach based on the analytical model, this lack of robustness would be a major problem and the internal resonances must be addressed.

More generally, since internal resonance is a physical mechanism for transferring vibration energy between modes, it is of interest to develop analytical methods for capturing this effect. One approach is to consider the specific case of internal resonance in the order $\varepsilon^{1}$ equations, imposing solvability conditions to obtain the modulation equations in the internal resonance case. Different methods might be utilized, but in either case, the internal resonance issue for the investigated chain is seen to be a rather "singular" phenomena, occurring for specific wavenumber/amplitude 
and material parameter combinations. Determining these combinations might be of interest, but transferring the results to a finite setting could be enough of a perturbation to suppress the internal resonance. This sensitivity means that the analysis of using internal resonance as a method of energy transfer is more suitable for specific structural systems rather than the constituent materials.

\section{Acknowledgements}

The authors were supported by ERC starting grant no. 279529 INNODYN. Furthermore, Niels M. M. Frandsen expresses his gratitude to Jon Juel Thomsen and Vladislav Sorokin for fruitful discussions on nonlinear dynamics.

[1] L. Brillouin. Wave Propagation in Periodic Structures. Dover Publications, 1953.

[2] C. Elachi. Waves in active and passive periodic structures: A review. Proc. IEEE, 64 (1976) 1666-1698.

[3] D.J. Mead. Wave propagation in continuous periodic structures: Research contributions from Southampton, 1964-1995. J. Sound Vib., 190(3) (1996) 495-524.

[4] M. I. Hussein, M. J. Leamy, and M. Ruzzene. Dynamics of Phononic Materials and Structures: Historical Origins, Recent Progress, and Future Outlook. Appl. Mech. Rev., 66(4) (2014) 040802 .

[5] S. Halkjær, O. Sigmund, and J. S. Jensen. Maximizing band gaps in plate structures. Struct. Multidiscip. O., 32(4) (2006) 263-275.

[6] G. Chakraborty and A. K. Mallik. Dynamics of a weakly non-linear periodic chain. Int. J. Non-Linear Mech., 36 (2001) 375-389.

[7] R. K. Narisetti, M. Ruzzene, and M. J. Leamy. A Perturbation Approach for Predicting Wave Propagation in One-Dimensional Nonlinear Periodic Structures. Trans. ASME, J. Vib. Acoust., 132 (2010) 1-11.

[8] R. K. Narisetti, M. Ruzzene, and M. J. Leamy. A perturbation approach for analyzing dispersion and group velocities in two-dimensional nonlinear periodic lattices. Trans. ASME, J. Vib. Acoust., 133 (2011) 1-12.

[9] K. L. Manktelow, M. J. Leamy, and M. Ruzzene. Multiple scales analysis of wave-wave interactions in a cubically nonlinear monoatomic chain. Nonlinear Dyn., 63 (2011) 193-203.

[10] N. Z. Swinteck, K. Muralidharan, and P. A. Deymier. Phonon scattering in one-dimensional anharmonic crystals and superlattices: Analytical and numerical study. J. Vib. Acoust., $135(4)$ (2013) 041016.

[11] K. L. Manktelow, M. J. Leamy, and M. Ruzzene. Weakly nonlinear wave interactions in multi-degree of freedom periodic structures. Wave Motion, 51 (2014) 886-904 .

[12] J. Cabaret, V. Tournat, and P. Béquin. Amplitude-dependent phononic processes in a diatomic granular chain in the weakly nonlinear regime. Phys. Rev. E, 86(4) (2012) 041305.

[13] B. Yousefzadeh and A. S. Phani. Energy transmission in finite dissipative nonlinear periodic structures from excitation within a stop band. J. Sound Vib., 354 (2015) 180-195.

[14] F. Bloch. Über die quantenmechanik der elektronen in kristalgittern. Z. Phys., 52 (1929) $555-600$.

[15] A. H. Nayfeh. Introduction to Perturbation Techniques. John Wiley and Sons, 1981. 


\section{Appendix A. Coefficient vectors}

The coefficient vectors from Eq. (10) are given by

$$
\begin{aligned}
\mathbf{q}_{1} & =-i 2 \omega_{1} \mathbf{M M}^{-1 / 2} a_{1}^{\prime} \mathbf{z}_{1}-3 a_{1}^{2} \bar{a}_{1} \sum_{l, m, n} \mathbf{h}_{l m n} z_{1}^{l} z_{1}^{m} \bar{z}_{1}^{n}-6 a_{1} a_{2} \bar{a}_{2} \sum_{l, m, n} \mathbf{h}_{l m n} z_{1}^{l} z_{2}^{m} \bar{z}_{2}^{n} \\
\mathbf{q}_{2} & =-i 2 \omega_{2} \mathbf{M M}^{-1 / 2} a_{2}^{\prime} \mathbf{z}_{2}-3 a_{2}^{2} \bar{a}_{2} \sum_{l, m, n} \mathbf{h}_{l m n} z_{2}^{l} z_{2}^{m} \bar{z}_{2}^{n}-6 a_{2} a_{1} \bar{a}_{1} \sum_{l, m, n} \mathbf{h}_{l m n} z_{2}^{l} z_{1}^{m} \bar{z}_{1}^{n} \\
\mathbf{q}_{12} & =-3 a_{1}^{2} \bar{a}_{2} \sum_{l, m, n} \mathbf{h}_{l m n} z_{1}^{l} z_{1}^{m} \bar{z}_{2}^{n} \quad, \quad \mathbf{q}_{21}=-3 \bar{a}_{1} a_{2}^{2} \sum_{l, m, n} \mathbf{h}_{l m n} z_{2}^{l} z_{2}^{m} \bar{z}_{1}^{n} \\
\mathbf{p}_{12} & =-3 a_{1}^{2} a_{2} \sum_{l, m, n} \mathbf{g}_{l m n} z_{1}^{l} z_{1}^{m} z_{2}^{n} \quad, \quad \mathbf{p}_{21}=-3 a_{1} a_{2}^{2} \sum_{l, m, n} \mathbf{g}_{l m n} z_{1}^{l} z_{2}^{m} z_{2}^{n} \\
\mathbf{p}_{1} & =-a_{1}^{3} \sum_{l, m, n} \mathbf{g}_{l m n} z_{1}^{l} z_{1}^{m} z_{1}^{n} \quad, \quad \mathbf{p}_{2}=-a_{2}^{3} \sum_{l, m, n} \mathbf{g}_{l m n} z_{2}^{l} z_{2}^{m} z_{2}^{n}
\end{aligned}
$$

The coefficient vectors $\mathbf{h}$ and $\mathbf{g}$ can be found in Tables A.3 and A.4 respectively.

Table A.3: Coeffecient vector $\mathbf{h}$

\begin{tabular}{rrr}
$l, m, n$ & $\mathbf{h}(1)\left[\frac{1}{m_{1} m_{2}}\right]$ & $\mathbf{h}(2)\left[\frac{1}{m_{1} m_{2}}\right]$ \\
\hline $1,1,1$ & $\frac{m_{2}}{\sqrt{m_{1}}}\left(\Gamma_{1}+\Gamma_{2}\right)$ & $-\frac{m_{2}}{\sqrt{m_{1}}}\left(\Gamma_{1}+\Gamma_{2} e^{i \mu}\right)$ \\
$1,1,2$ & $-\sqrt{m_{2}}\left(\Gamma_{1}+\Gamma_{2} e^{i \mu}\right)$ & $\sqrt{m_{2}}\left(\Gamma_{1}+\Gamma_{2} e^{2 i \mu}\right)$ \\
$1,2,1$ & $-\sqrt{m_{2}}\left(\Gamma_{1}+\Gamma_{2} e^{-i \mu}\right)$ & $\sqrt{m_{2}}\left(\Gamma_{1}+\Gamma_{2}\right)$ \\
$2,1,1$ & $-\sqrt{m_{2}}\left(\Gamma_{1}+\Gamma_{2} e^{-i \mu}\right)$ & $\sqrt{m_{2}}\left(\Gamma_{1}+\Gamma_{2}\right)$ \\
$1,2,2$ & $\sqrt{m_{1}}\left(\Gamma_{1}+\Gamma_{2}\right)$ & $-\sqrt{m_{1}}\left(\Gamma_{1}+\Gamma_{2} e^{i \mu}\right)$ \\
$2,1,2$ & $\sqrt{m_{1}}\left(\Gamma_{1}+\Gamma_{2}\right)$ & $-\sqrt{m_{1}}\left(\Gamma_{1}+\Gamma_{2} e^{i \mu}\right)$ \\
$2,2,1$ & $\sqrt{m_{1}}\left(\Gamma_{1}+\Gamma_{2} e^{-2 i \mu}\right)$ & $-\sqrt{m_{1}}\left(\Gamma_{1}+\Gamma_{2} e^{-i \mu}\right)$ \\
$2,2,2$ & $-\frac{m_{1}}{\sqrt{m_{2}}}\left(\Gamma_{1}+\Gamma_{2} e^{-i \mu}\right)$ & $\frac{m_{1}}{\sqrt{m_{2}}}\left(\Gamma_{1}+\Gamma_{2}\right)$ \\
&
\end{tabular}

Table A.4: Coeffecient vector $\mathbf{g}$

\begin{tabular}{rrr}
$l, m, n$ & $\mathbf{g}(1)\left[\frac{1}{m_{1} m_{2}}\right]$ & $\mathbf{g}(2)\left[\frac{1}{m_{1} m_{2}}\right]$ \\
\hline $1,1,1$ & $\frac{m_{2}}{\sqrt{m_{1}}}\left(\Gamma_{1}+\Gamma_{2}\right)$ & $-\frac{m_{2}}{\sqrt{m_{1}}}\left(\Gamma_{1}+\Gamma_{2} e^{3 i \mu}\right)$ \\
$1,1,2$ & $-\sqrt{m_{2}}\left(\Gamma_{1}+\Gamma_{2} e^{-i \mu}\right)$ & $\sqrt{m_{2}}\left(\Gamma_{1}+\Gamma_{2} e^{2 i \mu}\right)$ \\
$1,2,1$ & $-\sqrt{m_{2}}\left(\Gamma_{1}+\Gamma_{2} e^{-i \mu}\right)$ & $\sqrt{m_{2}}\left(\Gamma_{1}+\Gamma_{2} e^{2 i \mu}\right)$ \\
$2,1,1$ & $-\sqrt{m_{2}}\left(\Gamma_{1}+\Gamma_{2} e^{-i \mu}\right)$ & $\sqrt{m_{2}}\left(\Gamma_{1}+\Gamma_{2} e^{2 i \mu}\right)$ \\
$1,2,2$ & $\sqrt{m_{1}}\left(\Gamma_{1}+\Gamma_{2} e^{-2 i \mu}\right)$ & $-\sqrt{m_{1}}\left(\Gamma_{1}+\Gamma_{2} e^{i \mu}\right)$ \\
$2,1,2$ & $\sqrt{m_{1}}\left(\Gamma_{1}+\Gamma_{2} e^{-2 i \mu}\right)$ & $-\sqrt{m_{1}}\left(\Gamma_{1}+\Gamma_{2} e^{i \mu}\right)$ \\
$2,2,1$ & $\sqrt{m_{1}}\left(\Gamma_{1}+\Gamma_{2} e^{-2 i \mu}\right)$ & $-\sqrt{m_{1}}\left(\Gamma_{1}+\Gamma_{2} e^{i \mu}\right)$ \\
$2,2,2$ & $-\frac{m_{1}}{\sqrt{m_{2}}}\left(\Gamma_{1}+\Gamma_{2} e^{-3 i \mu}\right)$ & $\frac{m_{1}}{\sqrt{m_{2}}}\left(\Gamma_{1}+\Gamma_{2}\right)$ \\
& &
\end{tabular}

